\title{
Chest-Wall Metastasis of Prostate Cancer
}

\author{
Vlad Matei, M.D. ${ }^{2}$ and Sujata Bhushan, M.D. ${ }^{1}$ \\ 'Dallas VA Medical Center / UT Southwestern Medical Center, Dallas, TX, USA; ${ }^{2}$ UT Southwestern Medical Center, Dallas, TX, USA.
}

KEY WORDS: prostate cancer; metastasis; PSA.

J Gen Intern Med 30(12): 1888

DOI: $10.1007 / \mathrm{s} 11606-015-3270-1$

(C) Society of General Internal Medicine 2015

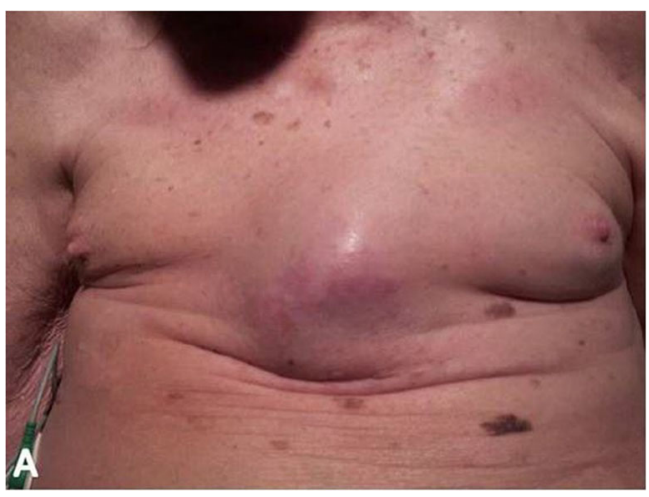

Panel A Showing the large anterior chest wall mass.

A n 88-year-old male presented with a gradually enlarging, painless mass on his anterior chest wall for 1 year (Panel A). On exam, there was a $12 \times 10 \times 5 \mathrm{~cm}$, nontender, firm, immobile mass over the body of the sternum. Computed tomography (CT) demonstrated a permeative chest-wall mass (Panel B). Serum prostate-specific antigen (PSA) was 3,019 ng/ml. Histopathology was consistent with prostate adenocarcinoma. The patient had poor functional status and requested hospice.

Chest wall tumors account for less than $1 \%$ of all neoplasms and can arise from either a soft tissue or bony structure. ${ }^{1}$ More than $50 \%$ of chest wall tumors are malignant. Malignant primaries include Ewing's sarcoma, osteosarcoma and chondrosarcoma. Malignant lesions tend to grow faster, are painful, and present as larger masses than benign lesions. Primary malignant tumors of the chest wall should be resected with wide margins and receive adjuvant therapy as appropriate. Metastatic disease accounts for $16 \%$ of malignant chest wall tumors. Chest wall

Received August 29, 2014

Revised November 24, 2014

Accepted February 24, 2015

Published online March 11, 2015

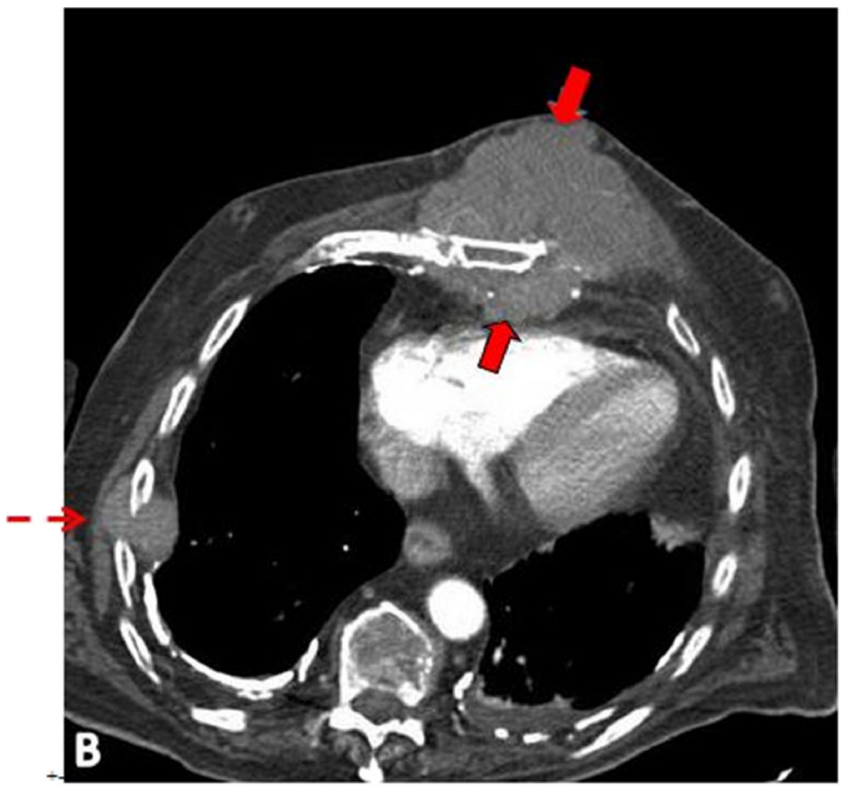

Panel B Computed tomography demonstrating a permeative chestwall mass (Panel B; between solid arrows) destroying the body of the sternum, abutting the pericardium, and indenting the anterior mediastinal fat. Rib lesion with a soft tissue component (Panel B; dashed arrow).

metastases occur more commonly from blood-borne spread than lymphatic or direct spread. The chest wall is an extremely rare site of prostate cancer metastasis. ${ }^{3}$ Case reports have described chest-wall metastasis regression with standard hormonal chemotherapy, with median survival of 12-24 months. ${ }^{2}$,

Corresponding Author: Sujata Bhushan, M.D.; Dallas VA Medical Center / UT Southwestern Medical Center, 4500 S Lancaster Road, Dallas, TX 75216, USA (e-mail: Sujata.bhushan@va.gov).

\section{REFERENCES}

1. David EA, Marshall M. Blair: Review of chest wall tumors: a diagnostic, therapeutic and reconstructive challenge. Semin Plast Surg. 2011;25:16-24.

2. Roxburgh CSD, Swami SK. Prostate cancer presenting as a chest wall tumour. Int J Urol. 2007;4:2

3. Wu JJ, Huang DB, Pang KR, Tyring SK. Cutaneous metastasis to the chest wall from prostate cancer. Int J Dermatol. 2006;45(8):946-8.

4. Chodak W, Keane T, Klotz L. Critical evaluation of hormonal therapy for carcinoma of the prostate. Urology. 2002;60:201. 